\title{
Study on Wireless Network Security in 4G Communication Technology
}

\author{
Jianbing Bai
}

Shaanxi Post and Telecommunication College, Xianyang, 712000, China

\author{
Keywords: 4G communication technology, Wireless network, Security Problem
}

\begin{abstract}
Wireless communication technology developed to $3 \mathrm{G}$ from early $1 \mathrm{G}$ and $2 \mathrm{G}$, and now it develops to $4 \mathrm{G}$ communication system direction. Relative to $1 \mathrm{G}, 2 \mathrm{G}$ and $3 \mathrm{G}, 4 \mathrm{G}$ communication technology improves greatly and owns openness and wireless transmission characteristics which make its application effect better. But since its system is more complex, wireless network security problem exists, which affects specific application of $4 \mathrm{G}$ communication technology. Thus, this paper analyzes and discusses wireless network security in 4G communication technology.
\end{abstract}

\section{Introduction}

Under rapid development of science and technology, 4G communication technology created based on science and previous development experience, is more superior to $3 G$ communication. Its operation speed, quality, sound effect and communication capability are strong. However, since wireless communication system is more complex and tedious, the application of 4G communication technology has security problem. It may threaten people's information security. Thus, during development of $4 \mathrm{G}$ communication technology, it is very significant to actively research and explore effective measures in order to handle security problems of 4G communication technology.

\section{Development history of wireless network communication mode}

With rapid development of science and technology, wireless network communication technology gain great progress. Seeing from development course of wireless network communication mode, wireless network communication mode mainly experiences the following stages.

\section{WPAN communication technology}

WPAN communication technology as the earliest wireless network communication mode is faced with the specific group, so its activity range is small. But its services are rich and diversified. Wireless network communication can be connected without seam and wire and offer wireless network for users. WPAN communication technology mainly includes two working modes: self-organizing network and basic structure mode. The implementation of two working modes can make WPAN play a role. Self-organizing network is connected with wireless terminal and can be communicated at will. This makes such working mode freer, so it can be applied flexibly. The working mode of basic structure network is that, wireless network forms through visiting node at wireless terminal so that users can communicate mutually. Such working mode makes communication quality of WPAN enhanced and improves security of user information. With the development of wireless communication technology, market demand for wireless communication technology increases so that the application of WPAN becomes increasingly frequent and exceeds its bearing capacity in quantity, communication distance and quality. Meanwhile, some problems appear to WPAN communication technology application. This promotes relevant researchers to actively study and innovate for wireless communication technology.

\section{GSM/GPRS communication technology}

GSM is a new communication technology after WPAN and is a typical example of 2G system. GSM communication technology is characterized by wide coverage area, low operation cost and strong reliability. Thus, it makes up for some defects of WPAN communication technology and improves application effect of wireless communication technology. GPRS is a kind of wireless data transmission service system. Its foundation is GSM, but differs a lot with GSM. It sends and receives 
data under terminal-to-terminal grouping and transfer mode, and it does not use network resource under circuit switching mode. GPS cannot reach this. Thus, GSM/GPRS communication technology has great innovations, compared with WPAN and meets people's demand for wireless communication to some extent. But, from the perspective of continuous development of wireless communication technology, GSM/GPRS communication technology has many defects. This is also one of causes why wireless communication technology develops continuously.

\section{G communication technology}

The application of $3 \mathrm{G}$ communication technology makes wireless communication quality, service, capacity and security greatly innovate. Relative to 2G communication technology, 3G communication technology connects wireless system with internet and adopts seamless roaming technology so that the capacity, communication quality, communication speed, communication function and communication security of 3G communication system improve greatly. Hence, 3G communication technology is widely applied. Although 3G communication technology owns many advantages, there are still many problems in actual operation process. For example, voice exchange architecture is not pure IP mode; the date transmitted at a high speed are not mature enough; there are too many security algorithms and they are not uniform enough; authentication protocol may be easily attacked.

\section{G communication technology}

Different from 3G communication technology, 4G communication technology achieves network convergence and fuses wireless communication system and technology so that $4 \mathrm{G}$ communication technology can meet users' more wireless network needs. 4G communication technology still applies TD-SCDMA frame structure and rationally applies TD-LTE technology, including link self-adaptation, multiple access technology and multi-antenna technology so that 4G communication technology is applied more efficiently ${ }^{[1]}$.

\section{Network structure and advantages of 4G communication technology}

4G communication technology integrates WLAN, bluetooth, honeycomb signal, honeycomb to satellite communications and wired network. Such structural system provides technical needs for broadband wireless connections. Thus, 4G communication network can effectively connect wireless communication and technology and offer people with a seamless mobile computing environment. Users may transmit data, image and language at a high speed under such environment. From the perspective of network structure of $4 \mathrm{G}$ communication technology, 4G communication technology has many advantages as follows:

a). Communication speed is faster. Because $4 \mathrm{G}$ communication technology utilizes multiple communication technologies and rich experience, 4G channel occupies about 100MHZ frequency spectrum and greatly increases the breadth of network frequency spectrum. Thus, 4G has faster communication speed.

b). System capacity is larger. 4G communication technology achieves network convergence, skillfully applies large network storage capacity, incre3ase capacity of $4 \mathrm{G}$ communication system. Thus, its coverage is wider.

c). Communication cost is cheaper. The functions of $4 \mathrm{G}$ communication technology increase, so multiple communication services may derive out so that $4 \mathrm{G}$ communication operation can gain profits from more aspects. Corresponding communication cost will properly reduce to attract vast users to apply $4 \mathrm{G}$ communication network.

d). Autonomy and self-adaptation are strong. 4G communication technology intensifies automatic management and makes automatic management functions stronger. To strictly control 4G communication operation, communication services in various aspects are required to reach corresponding standards. In this way, autonomy and self-adaptation of $4 \mathrm{G}$ communication technology are strong ${ }^{[2]}$. 


\section{Wireless network security in 4G communication technology}

Based on the above overview of $4 \mathrm{G}$ communication technology, $4 \mathrm{G}$ communication is a broadband communication system which integrates multiple functions. But its system is too complex and tedious, so $4 \mathrm{G}$ communication technology has some wireless network security problems as follows.

\section{Poor security of hardware platform of mobile terminal}

4G system belongs to a basic type of communication system. Its components include four parts: wireless core network, terminal, wireless access network and IP backbone network. There are many specific components of $4 \mathrm{G}$ communication system. Thus, many wireless network security problems will appear in specific operation process. The hardware platform of mobile terminal is one of the causes for wireless network security problems. At present, hardware platform of mobile terminal connected with 4G communication generally lacks authentication mechanism and protection mechanism, so some modules in hardware platform of mobile terminal may be easily tampered by invaders. Finally, the application of hardware platform of mobile terminal is serious influenced. Besides, communication interface in mobile terminal fails to form protection mechanism which integrates integrity and confidentiality, so security of mobile terminal is poor and it may be attacked by hacker to steal important information or damage mobile terminal. In such case, 4G communication system will certainly be influenced by mobile terminal, and its wireless network security will be threatened.

\section{Lack of operating system security}

Wireless network security of $4 \mathrm{G}$ communication system is related to operating system security. This is because the components of $4 \mathrm{G}$ communication system are many. This will inevitably cause mismatch of some components, influence $4 \mathrm{G}$ communication system operation and make its operating system have some loopholes. Because many aspects of 4G communication system are open, the loopholes also have openness characteristic, so the application of $4 \mathrm{G}$ communication system is doubted by users ${ }^{[3]}$. Thus, operating system security should be valued in the stage of rapid development of $4 \mathrm{G}$ communication technology. It is required to optimize operating system and boost application effect of $4 \mathrm{G}$ communication system.

\section{Loopholes of application program}

In actual application of 4G wireless system, mobile terminal can support multiple wireless application functions, such as email and ecommerce, and expand more wireless communication services. To effectively operate various communication services, corresponding application program needed to be formulated for each wireless application function supported by mobile terminal so that each wireless application function of mobile terminal can be fully exerted. But if application program of wireless application functions of mobile terminal have defects, some loopholes or potential safety hazards will appear in application of wireless application functions of mobile terminal. These loopholes or potential safety hazards will indirectly affect $4 \mathrm{G}$ communication system and threaten wireless network composition of 4G communication system. Hence, the defect of application program of wireless application functions of mobile terminal is one of the reasons for wireless network secueity problem of 4G communication system.

\section{Main strategies to improve 4G wireless communication network security}

In the face of communication network secueity of 4G wireless communication technology, effective measures should be taken to improve network security of $4 \mathrm{G}$ wireless communication and create conditions for applying 4G wireless communication technology.

\section{Research, develop and utilize reinforced operating system}

To evade wireless network security as far as possible, it is very necessary to optimize 4G communication system operation. Hence, it is required to study and utilize reinforced operating system. In other words, it is required to analyze operating system of $4 \mathrm{G}$ communication system, specify potential safety hazards of operating system, select operating system which meets TMP demand based on ensuring compatibility, security and security of $4 \mathrm{G}$ communication system, and 
guarantee long-distance verification, regional isolation and mixed access control operations of operating system so as to make reinforced operating system more applicable and boost application effect of $4 \mathrm{G}$ communication system ${ }^{[4]}$.

\section{Continuously reinforce hardware platform}

Aiming at the situation where hardware platform secueity of mobile terminal is not high, hardware platform should be regarded as the object of basic protection. It is required to detect and start hardware platform in an all-around way regularly or irregularly, master application conditions of hardware platform, check whether it has faults or defects, then effectively adjust and optimize it as well as boost applicability of hardware platform. Besides, suitable and effective protection measures should be adopted to intensify core network. TD-SCDMA is the core network of 4G wireless communication, though it is not influenced by symmetrical control. The development and implementation of TD-LTE have far exceeded TD-SCDMA. In such case, TD-LTE can replace TD-SCDMA. Hardware platform can be intensified for secure application through adding the total number of networks of $4 \mathrm{G}$ wireless communication.

\section{Improve secueity of wireless access network and mobile terminal}

To solve wireless network security problems of $4 \mathrm{G}$ communication technology, it is also required to focus on wireless access network and mobile terminal. The specific practice is as follows:

a). Identity authentication. To improve wireless network security of $4 \mathrm{G}$ communication system, two-directional identity authentication mechanism is established based on digital certificate through mobile terminal and wireless access network. It restrains identity authentication so that identity authentication can be executed strictly. Meanwhile, it avoids loopholes in identity authentication and invasion in mobile terminal.

b). Security access. Security access of wireless access network amounts to a security line of defense and can improve secueity of 4G communication system. To make wireless access network accessed safely, wireless access network needs to provide security access function of credible mobile terminal through auxiliary security equipment so as to be safely accessed in mobile terminal ${ }^{[5]}$.

\section{Conclusions}

As 4G communication technology is gradually popularized, wireless network security problem in 4G communication technology influences application effect of $4 \mathrm{G}$ communication technology to some extent and threatens people's information security. To effectively solve such problem, it is required to research, develop and utilize reinforced operating system, continuously reinforce hardware platform, and boost secueity of wireless access network and mobile terminal in order to greatly improve secueity of $4 \mathrm{G}$ communication network and create conditions for effective application of 4G communication technology. Thus, it is very necessary to effectively handle wireless network secueity in $4 \mathrm{G}$ communication technology.

\section{References}

[1] Dong Keke, Analysis of network security problem of communication technology and countermeasures. Heilongjiang Science and Technology Information, 2015(14):132-132.

[2] Dong Zhongliang, Exploration of wireless network secueity protection based on 4G communication technology. Super Science, 2015(7):274-275.

[3] Xie Jingxian, Innovation and development of 4G communication technology. Information Communication, 2012(4):207-208.

[4] Yao Kewei, Analysis and discussion of 4G communication technology. Silicon Valley, 2011(17):8-8.

[5] Mao Chao, Secure communication of wireless network of communication technology. Urban Construction Theory Research (Electronic Edition), 2015(4):2996-2996. 